\title{
Surface Magnetostriction Model for MagNEMS
}

\author{
T. Szumiata*, M. Gzik-Szumiata
}

Department of Physics, University of Technology and Humanities in Radom, Krasickiego 54, 26-600 Radom, Poland

\begin{abstract}
In this work the influence of surface roughness on magnetostrictive nano-actuator parameters has been analyzed theoretically. A mechanical and magnetoelastic behavior of investigated cantilever bimorphic system has been described in the frame of the simple analytical model. Realistic material parameters have been incorporated into the model for high-magnetostrictive galfenol $(\mathrm{Fe}-\mathrm{Ga})$ thin films on silicon substrate. It has been shown that for $5 \mathrm{~nm}$ thick galfenol film a flat surface magnetostrictive effects modify the cantilever deflection and force only by $3 \%$, whereas in the case of rough surface this influence increases to about $15 \%$, when dimensions of roughness steps are comparable to the distances between them.
\end{abstract}

DOI: $10.12693 /$ APhysPolA.126.200

PACS: $62.25 .-\mathrm{g}, 85.85 .+\mathrm{j}, 75.80 .+\mathrm{q}, 85.70 . \mathrm{Ec}, 68.35 .-\mathrm{p}$

\section{Introduction}

During last two decades a significant technological progress in the field of magnetic microelectromechanical systems (MagMEMS), as well as the increase of application potential of such systems, has been observed [1]. Typical dimensions of magnetostrictive cantilever-type MagMEMS are: thickness of magnetostrictive layer of several $\mu \mathrm{m}$, thickness of substrate - several tens of $\mu \mathrm{m}$, total width of hundreds of $\mu \mathrm{m}$ and total length - of the order of $\mathrm{mm}$. Next technological step in the miniaturization is the transition to nanoscale - from MEMS to nanoelectro-mechanical systems (NEMS) devices [2]. It is a direct way towards designing of nanosensors and nanoactuators (e.g. motors, pumps and valves) of unique parameters (high frequency operation, low energy consumption) and for their integration with nanoelectronics. In such systems the low-dimensional effects (quantum, surface and thermal ones) significantly affect their properties. The main goal of present work was to investigate theoretically a surface roughness influence on parameters of magnetostrictive bimorphic nano-actuator. The estimation of nano-cantilever deflection and force has been made for the real system of high-magnetostrictive galfenol nanofilm $[7,8]$ deposited on thin silicon substrate.

\section{Theoretical model}

Bimorphic nano-actuator consisting of magnetostrictive layer, placed on nonmagnetic thin base, has been considered as an object of this study. A mechanical and magnetoelastic behavior of investigated cantilever bimorphic system has been described in the frame of the simple analytical model proposed by Ishiyama and Yakota [3]. This relatively new model (2008) is a good alternative to the old Trémolet de Lacheisserie analytical formulae (1996) [4], since it considers a dependence of neutral plane position of bimorphic system on elastic constants. Moreover, it is more convenient and universal

*corresponding author; e-mail: t.szumiata@uthrad.pl

than numerical finite elements method (FEM) models [5]. Within model [3] the deflection $\delta$ of nano-cantilever and generated force $F$ are described by following formulae:

$$
\begin{aligned}
& \delta=\frac{\frac{3}{4} L^{2}\left[E_{s} \lambda_{s u b} t_{s}\left(-t_{s}+2 t_{s}^{\prime}\right)+E_{f} \lambda_{f} t_{f}\left(t_{f}+2 t_{s}^{\prime}\right)\right]}{E_{s} t_{s}\left(t_{s}^{2}-3 t_{s} t_{s}^{\prime}+3 t_{s}^{\prime 2}\right)+E_{f} t_{f}\left(t_{f}^{2}+3 t_{f} t_{s}^{\prime}+3 t_{s}^{\prime 2}\right)}, \\
& t_{s}^{\prime}=\frac{E_{s} t_{s}^{2}-E_{f} t_{f}^{2}}{2\left(E_{s} t_{s}+E_{f} t_{f}\right)}, \\
& F=\frac{3 w}{4 L}\left[E_{s} \lambda_{s u b} t_{s}\left(-t_{s}+2 t_{s}^{\prime}\right)+E_{f} \lambda_{f} t_{f}\left(t_{f}+2 t_{s}^{\prime}\right)\right],
\end{aligned}
$$

where $L$ and $w$ are the cantilever length and width, respectively; $t_{f}, E_{f}$, and $\lambda_{f}$ are the thickness, Young's modulus, and the magnetostriction constant of the magnetic thin film, respectively; $t_{s}, E_{s}$, and $\lambda_{s u b}$ are the thickness, Young's modulus, and possible magnetostriction constant of the substrate, respectively; $t_{s}^{\prime}$ is the distance between the neutral plane and contact plane.

a)

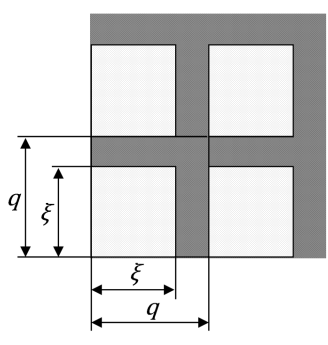

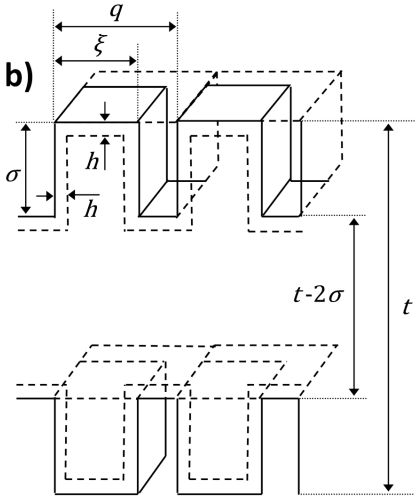

Fig. 1. Scheme of the film roughness model: a) projection from the top, $b$ ) a perspective view of the surface [6].

Magnetostriction changes, due to the presence of surface roughness (Fig. 1) of magnetostrictive layer, has been considered in model developed in [6]. The following formulae:

$$
\lambda_{f}=\lambda^{v}+\lambda^{\operatorname{surf}} \cdot \frac{2}{t_{f}},
$$




$$
\lambda^{\text {surf }}=\left(\lambda^{s}-\lambda^{v}\right) \cdot h \cdot\left(1+\frac{4 \sigma \xi}{q^{2}}\right),
$$

describe the effective saturation magnetostriction constant $\lambda_{f}$ of magnetostrictive layer as a function of its thickness $t_{f} ; \lambda^{v}$ is a magnetostriction constant of bulk material, $\lambda^{s}$ - magnetostriction constant of flat surface region, $h$ - thickness of surface region (half of lattice constant); $\sigma$ - depth of cubicoidal roughness steps; $\xi$ - inplane size of steps repeated with period $q$. Within pseudodipolar exchange interactions model for bcc crystalline structure [6] a surface magnetostriction constant is equal $\lambda^{s}=\lambda^{v} / 2$.
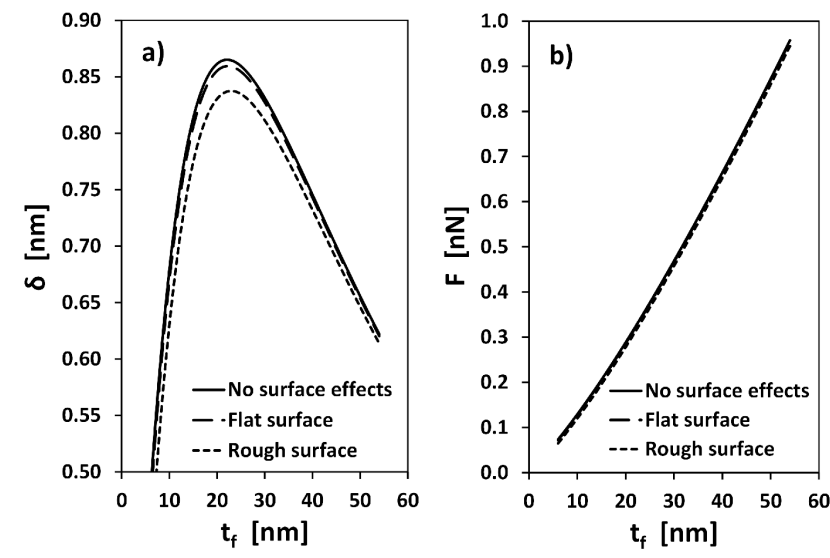

Fig. 2. Nano-cantilever a) deflection and b) force as a function of magnetostrictive layer thickness for different surfaces.
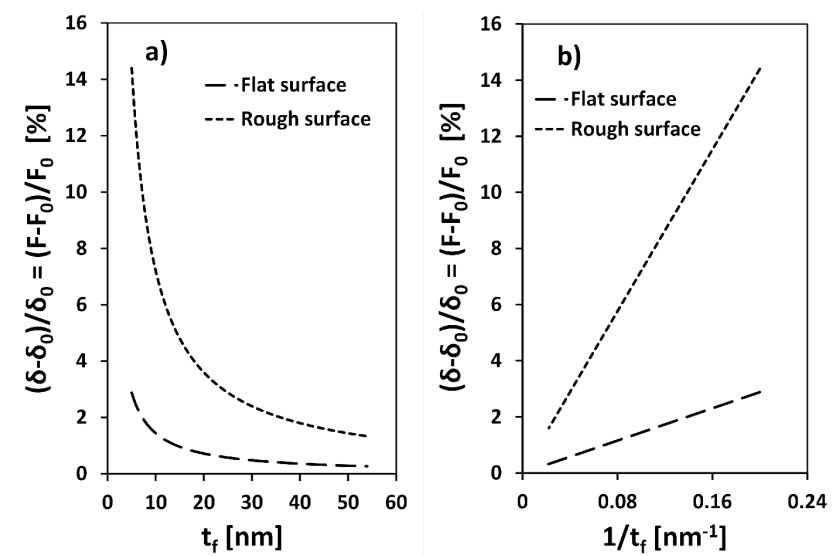

Fig. 3. Relative deflection and force of nano-cantilever versus a) magnetostrictive layer thickness and b) its reciprocal - for flat and rough surfaces (quantities with subscript "0" refer to the case without any surface effects).

\section{Results}

Realistic material parameters have been incorporated into the model. High-magnetostrictive galfenol (Fe-Ga) thin films on silicon substrate has been considered [7, 8]. Magnetostriction and elastic constants both for layer and substrate have been taken from literature [8-10] (for silicon the magnetostriction has been assumed to be zero). The length and width of cantilever has been fixed as $1000 \mathrm{~nm}$ and $100 \mathrm{~nm}$, respectively, whereas the thickness of the substrate - as $30 \mathrm{~nm}$. The dimensions of roughness steps have been assumed to be comparable to the distance between them. Absolute and relative deflection of the actuator and generated force are presented in Fig. 2 and Fig.3, respectively, as a function of galfenol film thickness. It is demonstrated that e.g. for $5 \mathrm{~nm}$ thick galfenol film a flat surface magnetostrictive effects modify the cantilever deflection only by $3 \%$, whereas in the case of rough surface this influence increases to about $15 \%$. The absolute deflection seems to be almost not sensitive to the magnetostrictive surface effects, however the relative one depends similarly to the relative deflection change, i.e. increases linearly with reciprocal of the thickness. Moreover the roughness causes a shift of the optimal layer thickness corresponding to the maximal deflection.

\section{Conclusions}

As has been derived, for bimorphic magnetostrictive actuator of nanometric size based on galfenol the total deflections is a fraction of nanometers, and generated force - a fraction of nanonewtons. The presence of surface roughness magnifies several times the influence of surface magnetostriction effects on the mechanical parameters of the actuator. In real systems this influence could be even stronger due to the possible surface porosity at atomic scale.

\section{References}

[1] M.R.J. Gibbs, J. Magn. Magn. Mater. 290-291, 1298 (2005).

[2] C.T. Leondes, MEMS/NEMS - Handbook Techniques and Applications, Spinger 2006.

[3] K. Ishiyama, Ch. Yokota, J. Magn. Magn. Mater. 320, 2481 (2008).

[4] E. du Trémolet de Lacheisserie, J.C. Peuzin, J. Magn. Magn. Mater. 136, 189 (1994).

[5] R. Watts, M.R.J. Gibbs, W.J. Karl, H. Szymczak, Appl. Phys. Lett. 70, 2607 (1997).

[6] T. Szumiata, M. Gzik-Szumiata, K. Brzózka, Materials Science - Poland 26, 1039 (2008).

[7] A. Javed, N.A. Morley, M.R.J. Gibbs, J. Magn. Magn. Mater. 321, 2877 (2009).

[8] A. Javed, N.A. Morley, T. Szumiata, M.R.J. Gibbs, Appl. Surf. Sci. 257, 5977 (2011).

[9] M.A. Hopcroft, W.D. Nix, T.W. Kenny, IEEE J. of Microelectromech. Syst. 19, 229 (2010).

[10] H.M. Schurter, A.B. Flatau, Proc. SPIE 6929, Behavior and Mechanics of Multifunctional and Composite Materials $2008 \mathbf{6 9 2 9 1 U}$ (April 02, 2008). 